\title{
PRUEBA JUDICIAL Y PRÁCTICA DEL DISCOVERY \\ EN LA UNIÓN EUROPEA
}

Gloria Esteban de la Rosa

Profesora Titular de Universidad de Derecho internacional privado

Universidad de Jaén

E-mail: gesteban@ujaen.es

Resumen: La obtención de pruebas en el extranjero plantea cuestiones cada vez más complejas, derivadas de la diversidad de términos y expresiones que se emplean en cada uno de los ordenamientos de los países de la Unión Europea en el campo del Derecho procesal, así como de las concepciones sobre la función y finalidad del procedimiento. Dicha diversidad se manifiesta en la propia concepción de la prueba, en la medida en que en algunos sistemas jurídicos existe el procedimiento de discovery, cuya compatibilidad con las reglas que regulan la instrucción en los ordenamientos pertenecientes a la tradición civilista se ha puesto en tela de juicio.

Palabras clave: obtención de pruebas en el extranjero, discovery, espacio judicial europeo

\begin{abstract}
It's necessary to know when it is possible to use the Regulation 1206/2001 for obtaining evidences in the territory of one State member of the European Union when it is required to use the discovery by other. We can find already decisions of the European Justice Court that can be followed to clarify this possibility. There are very well known the problems that posed the Hague Convention of 1970 between the judicial authorities of United States of American when they wanted to obtain evidences in Europe through discovery.
\end{abstract}

Keywords: discovery, taking of evidence abroad, European judicial space 

EXTRANJERO

\title{
1.1. El Reglamento 1206/2001/CE del Consejo, de 28 de mayo de 2001
}

\author{
A) Finalidad
}

El Reglamento 1206/2001/CE del Consejo, de 28 de mayo de 200, relativo a la cooperación entre los órganos jurisdiccionales de los Estados miembros en el ámbito de la obtención de pruebas en materia civil y mercantil, permite la práctica de diligencias de obtención de pruebas en el espacio europeo ${ }^{1}$. No se refiere ni a los medios y a la actividad de instrucción, sino que establece vías de colaboración entre los órganos judiciales de los Estados parte de la UE para la práctica u obtención de pruebas en materia civil y mercantil ${ }^{2}$.

\footnotetext{
${ }^{1}$ DOCE, Serie L, núm. 174, de 27 de junio de 2001. Su génesis se encuentra en una Iniciativa de la República Federal alemana con vistas a la adopción de un Reglamento del Consejo, relativo a la cooperación entre los órganos jurisdiccionales de los Estados miembros en el ámbito de la obtención de pruebas en el extranjero en materia civil y mercantil (DOCE, Serie C, núm. 314, de 3 de noviembre de 2000). Para un estudio de la norma comunitaria vide, entre otros, Herrera Petrus, CH., La obtención internacional de pruebas. Asistencia jurisdiccional en Europa. Studia Albornotiana, Pub. del RCE, Bolonia, 2005; DIAGO DIAGO, P., La obtención de pruebas en la Unión Europea, Aranzadi, Pamplona, 2003; FrIGO, M./Fumagalu, L, L'assistenza giudiziaria internazionale in materia civile, Notificazione, Assunzione di prove, Informazioni sul diritto straniero, Cedam, Padova, 2003, pp. 101 y ss; TrockER, N., "Note sul Regolamento n. 1206/2001 relativo all'assunzione delle prove in materia civile o commerciale", RDI, 2003-3, pp. 267 y ss; FUMAGALLI, L., "La nuova disciplina comunitaria dell'assunzione delle prove all'estero in materia civile", RDIPP, 2004, pp. 327 y ss; GIOIA, G., "Cooperazione fran autorità giudiziarie degli stati CE nell'assunzione delle prove in materia civile e commerciale", NLCC, 2001, pp. 1159 y ss; BONATTI, R., "Sovranità nazionale e leggi processuali nell'armonizzazione del diritto delle prove in Europa", RTDPC, 2004-I, pp. 211 y ss.

${ }^{2}$ De otra parte, se excluyen del instrumento comunitario las actuaciones y actos procesales, para los que se solicite la asistencia judicial, que no tengan una finalidad de instrucción, sino cautelar o ejecutiva. Véase, FUMAGALII, L., "La nuova disciplina comunitaria dell'assunzione delle prove all'estero in materia civile", RDIPP, 2002, núm. 2, p. 332.
} 


\section{Doctrina y Jurisprudencia}

Tal colaboración tiene lugar de dos formas: a través de la comunicación entre las autoridades judiciales de los Estados miembros, o de forma directa. De otra parte, prevé que dicha cooperación tenga lugar a través de los medios o instrumentos de instrucción existente en el Estado requerido, en el que se encuentra la materia o tema de prueba, salvo para el caso de que se obtenga de forma directa ${ }^{3}$.

Así, se trata de agilizar la práctica de pruebas en el territorio de un Estado comunitario distinto del lugar en el que se sigue el procedimiento de fondo (o se prevé incoar), de un lado; y, de otro, limitar los motivos por los que puede denegarse la solicitud ${ }^{4}$. Entró en vigor el 1 de julio de 2001, pero su art. 24 estableció dos momentos temporales para su aplicación, con la finalidad de que los Estados miembros de la UE pudieran preparar los medios necesarios para asegurar la efectividad de la cooperación internacional que instauraba ${ }^{5}$.

En cuanto a España, los órganos competentes a que se refiere el art. 2, $2^{\circ}$ del Reglamento 1206/2001 son los Juzgados de Primera Instancia de cada partido judicial mediante reparto a través del correspondiente Juez Decano. Por su parte, el órgano central designado por España (de conformidad con el art. 3) es la Subdirección General de Cooperación jurídica internacional del Ministerio de Justicia. De otro lado, las peticiones deberán dirigirse en castellano o portugués y el medio de comunicación que

\footnotetext{
${ }^{3}$ Se trata ésta, sin duda, de otras de las principales aportaciones del texto comunitario, que prevé en su art. 17, que es interpretado por la doctrina como un abandono del principio de territorialidad de lo actividad de instrucción, vinculado al principio de soberanía. Véase, LEBEAU, D./NIBOYET, M.L., "Regards croisés du processualiste et de l'internationaliste sur le Règlement CE, du 28 mai 2001, relatif á l'obtention des preuves civiles á l'étranger", Gaz. Pal., enero-febrero 2003, p. 222.

${ }^{4}$ Véase en este sentido, entre otros, LEBEAU, D./NiBoYeT, M.L., "Regards croisés du processualiste et de I'internationaliste sur le Règlement CE, du 28 mai 2001, relatif á l'obtention des preuves civiles á l'étranger", Gaz. Pal., enero-febrero 2003, p. 221.

${ }^{5}$ Así, los arts. 14, 19, 21 y 22 se aplican desde el 1 de julio de 2001, y desde el 1 de enero de 2004 la totalidad de sus disposiciones.
} 


\section{Doctrina y Jurisprudencia}

se acepta es el correo postal ${ }^{6}$. Por último, el art. 21 del Reglamento 1206/2001 deja abierta la posibilidad de utilizar otras vías, que puedan negociar los Estados miembros (entre ellas, la vía diplomática).

B) Ámbito de aplicación

La delimitación del ámbito material del Reglamento 1206/2001 requiere resolver previamente algunas cuestiones calificatorias, que no son de importancia menor, dada la estrecha relación entre la concepcion civil y procesal de la prueba ${ }^{7}$. Ahora bien, queda claro que se ocupa de la segunda. De otro lado, la expresión "prueba" es utilizada intencionadamente de forma imprecisa para proporcionar - en la medida de lo posibleun marco común entre los Estados comunitarios, de tal manera que esta noción comprenda cualquier estructura de prueba reconocida por los dos Estados miembros, que participan en su obtención en el extranjero ${ }^{8}$.

Relacionada con esta cuestión se sitúa la definición de la materia civil y mercantil. En concreto, cabe preguntarse si comprende la material laboral. Y, la respuesta ha de ser

\footnotetext{
${ }^{6}$ Véase la Guía Práctica para la aplicación del Reglamento relativo a las diligencias de obtención de pruebas, elaborada por los servicios de la Comisión de la UE en consulta con la Red Judicial Europea en materia civil y mercantil (http:europa.eu.int/civiljustice).

7 Es sabido que el Derecho de la prueba se encuentra "a caballo" entre el Derecho sustantivo y el procesal. Para el desarrollo de esta cuestión véase, CAMPEIS, G./DE PAULI, A., II processo civile italiano e lo straniero. Lineamenti di Diritto processuale civile internazionale, 2a ed., Giuffrè, 1996, que realizan detenidas consideraciones y reflexiones sobre la calificación de los distintos aspectos que presenta la prueba.

${ }^{8}$ Se trata de una noción de prueba, que permita ser solicitada por uno de los Estados miembros y aceptada por el otro, sin que el legislador comunitario haya querido tomar posición sobre las diferencias existentes entre pruebas típicas y atípicas, o entre prueba libre o prueba tasada. Tampoco ha querido el Reglamento 1206/2001 adentrarse en el concepto de prueba notoria o en el comportamiento concluyente por la actitud mantenida por las partes (véase, GIOIA, G., “Cooperazione...”, loc. cit., p. 1182).
} 


\section{Doctrina y Jurisprudencia}

positiva $^{9}$. En todo caso, está incluido el Derecho de familia ${ }^{10}$. También puede plantear ciertas dudas cuando se trata, p.ej., de decidir si queda comprendido el Derecho de Defensa de la competencia, al tratarse de un campo en el que la práctica de prueba puede estar relacionada con intereses de marcado carácter público e importancia económica $^{11}$. Ahora bien, conviene hacer una interpretación amplia de las materias que comprende la norma comunitaria, como ha señalado la Comisión ${ }^{12}$. En todo caso, queda excluida la materia penal o criminal ${ }^{13}$.

En segundo extremo, el art. 1, $2^{\circ}$ del Reglamento 1206/2001 limita su aplicación a las pruebas que se soliciten cuando se haya iniciado el procedimiento en el Estado requerido o se prevea incoar ${ }^{14}$. Esta fórmula pretende evitar que instrumentos relacionados con la obtención de pruebas destinadas a ser integradas en un proceso judicial sean utilizados con una finalidad policial.

\footnotetext{
9 Véase en este sentido, LeBEAU, D./NiBOYET, M.L., "Regards croisés du processualiste et de I'internationaliste sur le Règlement CE, du 28 mai 2001, relatif á l'obtention des preuves civiles á l'étranger", Gaz. Pal., enero-febrero 2003, p. 223.

${ }^{10}$ Ahora bien, el primer Informe preparado por la Comisión de la UE sobre la aplicación del Reglamento pone de relieve que se han suscitado dudas con respecto a la interpretación de la noción de prueba en el campo del Derecho de familia, especialmente en lo que se refiere a la obtención de muestras de ADN y muestras de sangre, en relación con los litigios suscitados en el ámbito de la filiación; así como pruebas periciales en materia de familia o bienestar infantil. Véase, "Informe de la Comisión... ", loc. cit., p. 6. También ha planteado esta mima duda la doctrina, indicando que hubiera sido necesario que el Reglamento indicase de forma más precisa su ámbito material (véase, GıolA, G., “Cooperazione...", loc. cit., p. 1169).

${ }^{11}$ Véase, TAVASSI, M./ SCUfFI, M., Diritto processuale antitrust, Milano, 2000.

${ }^{12}$ Véase, “Informe de la Comisión...", loc. cit., p. 6.

${ }^{13}$ Puede plantear también ciertas dudas la aplicación del Reglamento en el ámbito societario, en el que se está imponiendo -de forma cada vez más importante-la obligación de dar a conocer las cuentas. Véase, Stolowy, N., "De nouvelles injonctions en matière de publication des comptes par les sociétés", La Semaine Juridique, 22 février 2006, num. 8, pp. 114 y ss.

${ }^{14}$ Más concretamente, señala que: "no se solicitará la obtención de pruebas que no estén destinadas a utilizarse en una causa iniciada o que se prevea incoar". Y, por su parte, el art. 1, 2ㅇ del CLH de 1970 indica que: "no se empleará una comisión rogatoria para obtener pruebas que no estén destinadas a utilizarse en un procedimiento ya incoado o futuro".
} 
Así, suscita cierta complejidad precisar si quedan comprendidas en el ámbito del Reglamento las medidas de prueba, que se adoptan con una finalidad preparatoria del proceso de fondo ${ }^{15}$. Una de estas medidas son las diligencias preliminares, que, en realidad, no tienen una finalidad probatoria, sino que se practican con el fin de que el juez se cerciore de determinados extremos del proceso ${ }^{16}$. El hecho de que el Reglamento 1206/2001 sólo se refiera a la práctica de diligencia de obtención de pruebas, pero no incluya -a diferencia del Convenio de La Haya, de 18 de marzo de 1970- otras actuaciones judiciales, permite pensar que no comprende este tipo de medidas, pero sí el texto de La Haya de 1970 (véase infra) ${ }^{17}$.

De otro lado, surgen dudas cuando se trata de determinar si el Reglamento 1206/2001 incluye las medidas de aseguramiento de la prueba, que requieren una actividad procesal diferenciada de la que consiste en la anticipación de la prueba ${ }^{18}$. En

\footnotetext{
${ }^{15}$ Es sabido que los distintos ordenamientos prevén tipos de prueba (p.ej., pruebas preliminares), que permiten a la parte asegurarse de que no desaparecen los materiales que le pueden permitir incoar un proceso. En el caso del Derecho italiano, se trata de los "medios de instrucción preventiva". Véase, CONTE, M., "Cap. 23. I Mezzi di istruzzione preventiva» en, Le prove civile, Giuffrè Ed., Milano, 2005, pp. 597 y ss.

${ }^{16}$ Véase, Álvarez Alarcón, A., Las diligencias preliminares en el proceso civil, J.Ma Bosch Ed., Barcelona, 1997.

${ }^{17}$ A diferencia del Reglamento, el art. 1 del CLH de 1970 indica que: "en materia civil o mercantil, la autoridad judicial de un Estado contratante podrá, en conformidad a las disposiciones de su legislación, solicitar, de la autoridad competente de otro Estado contratante, por comisión rogatoria, la obtención de pruebas, así como la realización de otras actuaciones judiciales".

${ }^{18}$ La doctrina considera que la expresión que recoge el art. 1, 20 del Reglamento 1206/2001 ha de ser entendida en el sentido de que comprende las medidas de instrucción preventiva: testimonios a futura memoria, inspecciones judiciales, comprobaciones técnicas, etc., con exclusión de los procedimientos cautelares ante causam (véase, CAMPEIS, G./DE PAULI, A., "Prime riflessioni sul Regolamento sulle rogatorie : il nuovo assetto dell'assunzione di prove all'estero nel processo italiano", Giustizia civile, 2003, parte segunda, vol. III, p. 36). En todo caso, el instrumento comunitario se refiere a la práctica de prueba, debiendo interpretarse esta expresión en el sentido de la obtención del medio de prueba. Sin embargo, en el caso de las medidas de aseguramiento de la prueba, no se trata de la obtención del medio, sino de la conservación y custodia de las fuentes de prueba. Véase, Rizo GómEz, B., La anticipación de la prueba en el proceso civil, Titant lo Blanch, Valencia, 2010, p. 61.
} 


\title{
Doctrina y Jurisprudencia
}

ocasiones, la práctica de medidas de aseguramiento de la prueba también puede tener una finalidad de garantía $\mathrm{y}$, por ello, podría considerarse que se trata de medidas comprendidas en el art. 31 del Reglamento 44/2001/CE ${ }^{19}$.

Por último, hay que realizar una interpretación amplia del ámbito material del Reglamento 1206/2001, que no ha pretendido entrar en el fondo, ni del Derecho probatorio ni de los procedimientos de instrucción existentes en los ordenamientos de los Estados parte de la UE, sino que -tan sólo- ha tratado de facilitar la obtención de pruebas en el espacio comunitario, a través de la cooperación entre sus autoridades judiciales y su obtención directa (art. 17) ${ }^{20}$.

\subsection{EI Convenio de La Haya de 18 de marzo de 1970}

\author{
A) Génesis histórica
}

La preparación y negociación del Convenio de La Haya, de 18 de marzo de 1970, relativo a la obtención de pruebas en el extranjero en materia civil o mercantil (en adelante, CLH de 1970), tuvo lugar durante la Sesión 11 a de La Conferencia de La Haya

\footnotetext{
19 Ahora bien, no ha de perderse de vista que tienen, en todo caso, finalidad preparatoria de un procedimiento y que tratan de asegurar la desaparición de la prueba, no el cumplimiento de la sentencia de fondo. La doctrina italiana considera que la norma comunitaria comprende los "mezzi di istruzione preventiva", pero no los "procedimenti cautelari ante causam". Véase, CoNTE, M., "Cap. 23. I Mezzi... ", loc. cit., pp. 597 y ss.

${ }^{20}$ Ésta es la opinión de la Comisión de la UE, en el Primer Informe elaborado sobre la aplicación del Reglamento 1206/2001, de 5 de diciembre de 2007 [Documentos $\operatorname{COM(2007)} 769$ final, p. 6]. Ahora bien, ha de llamarse la atención sobre el hecho de que el Parlamento Europeo considerase insuficiente el contenido del referido Informe, que da una imagen equivocada con respecto a la mejora general en la aplicación del Reglamento 1206/2001 en el espacio comunitario. En concreto, el PE considera la Comisión ha de aportar ayuda concreta a los Estados, en el marco de la Estrategia e-Justice. Véase, Resolución del Parlamento Europeo, de 10 de marzo de 2009, sobre la cooperación entre las jurisdicciones de los Estados miembros, en el ámbito de la obtención de pruebas en materia civil y comercial [2008/2180(INI)], P6_TA (2009)0089.
} 


\section{Doctrina y Jurisprudencia}

de Derecho Internacional Privado, que se celebró del 7 al 28 de octubre de 1968, con el objetivo de realizar una revisión de los convenios existentes en el ámbito del procedimiento $^{21}$. Se introdujo la figura de las Autoridades Centrales (en adelante, AACC), con la finalidad de agilizar la tramitación de las comisiones rogatorias (art. 2). Estos organismos cumplen la función de recepción de la solicitud, siendo libre cada Estado de determinar la forma y la vía de transmisión de la comisión rogatoria ${ }^{22}$.

Ahora bien, el convenio también prevé que la prueba se practique por las autoridades diplomáticas o consulares de un Estado contratante, con respecto a sus propios nacionales, siempre que se refieran a un procedimiento incoado ante un Tribunal de dicho Estado y no se empleen medios coactivos (arts. 15 y ss). Y, por último, la obtención de pruebas en el extranjero también podrá tener lugar por un comisionado o comisario (abogado, juez o cualquier otra persona), siempre que se realicen sin compulsión, y se refieran a un procedimiento incoado ante un tribual de otro Estado contratante ${ }^{23}$.

Por último, se suscitaron una gran cantidad de dudas durante la elaboración del texto convencional con respecto al procedimiento de discovery, que condujeron a incluir en el art. 33 del convenio la posibilidad de realizar una reserva, para impedir que pudiese emplearse la cooperación internacional que instauraba el CLH en determinados casos de

\footnotetext{
${ }^{21}$ BOE núm. 203, de 25 de agosto de 1987. Para ello, se constituyó una Comisión, que elaboró un Ante-proyecto, que fue presentado, la undécima sesión de La Conferencia, con in Informe de Ph. W. Amram (véase, BATIFFOL, H., "La onzième session de la Conférence de La Haye de droit international privé», RCDIP, 1969, p. 16).

${ }^{22}$ Para el caso de España, la AC encargada de la recepción así como de la transmisión de las solicitudes de práctica de prueba es la Secretaria General Técnica del Ministerio de Justicia.

${ }^{23}$ El art. 16 del texto convencional también permite que los cónsules y personal diplomático practiquen pruebas en relación con personas que no sean nacionales del Estado que los envía. Pero, en tal caso, será necesario contar con la autorización del Estado huésped, no podrán emplearse medidas coactivas y el procedimiento de fondo ha de haberse incoado antes de que se practique la prueba en el extranjero.
} 


\section{Doctrina y Jurisprudencia}

discovery ${ }^{24}$. Tales dudas se presentaron por la diversa comprensión de este procedimiento en los países del common law y, en concreto, en el caso británico y americano, de un lado; y, de otro, por las dificultades que también tuvieron lugar para la interpretación de la propuesta de la Delegación británica (véase infra).

B) Ámbito de aplicación

Se aprecia la diferencia entre el ámbito de aplicación del Reglamento 1206/2001 y del CLH de 1970, en la medida en que se ha incluido una referencia expresa en este segundo a: "otras actuaciones judiciales", lo que plantea dudas con respecto a su interpretación, que ya se trataron durante su negociación ${ }^{25}$. Por tanto, el CLH de 1970, a diferencia del Reglamento 1206/2001, no sólo comprende las diligencias de obtención de prueba, sino también "otras actuaciones judiciales" (art. 1, 30), lo que permite considerar que su ámbito material es más amplio ${ }^{26}$.

De otro lado, cabe realizar una interpretación amplia del ámbito de aplicación del Convenio, siguiendo la misma línea que en los convenios anteriores, a los que sustituye $^{27}$. En concreto, esta expresión permite incluir las denominadas "diligencias

\footnotetext{
${ }^{24}$ Véase, entre otros, CARTIER, J.H., "Existing Rules and Procedures", Int. L., 1979, vol. 13, pp. 5 y ss; DeVINE, S./OLSEN, CH. M., "Taking Evidence outside of the United States", Boston University Law Review, 1975 , vol. 55, pp. 368 y ss.

${ }^{25}$ En concreto, se refería a la posibilidad de obtener muestras de sangre, certificados médicos o realizar un acto de conciliación, entre otros. Véase, AMRAM, PH. W., "Rapport de la Commission spéciale», Actes et documents de La Conférence de la Haye de Droit international privé, de la onzième session (7 au 26 octobre 1968), Tome IV. Obtention des preuves á l'étranger, La Haye, 1970, p. 55.

${ }^{26}$ Así, el art. 1 del citado Convenio dispone: "en materia civil o mercantil la autoridad judicial de un Estado contratante, podrá, en conformidad a las disposiciones de su legislación, solicitar de la autoridad competente de otro Estado contratante, por comisión rogatoria, la obtención de pruebas, así como la realización de otras actuaciones judiciales".

${ }^{27}$ Véase, EDWARD, D.M., “Taking of Evidence...", loc. cit., p. 647.
} 


\section{Doctrina y Jurisprudencia}

preliminares. Sin embargo, en todo caso, la expresión "otras actuaciones judiciales" no comprende ni la notificación de documentos judiciales ni las medidas de conservación o de ejecución (art. 1 , inciso $3^{\circ}$ ).

De otro lado, no se solicitará una comisión rogatoria para obtener pruebas que no estén destinadas a utilizarse en un procedimiento ya incoado o futuro (art. 1, 2 ${ }^{\circ}$ ). También en este caso cabe observar una diferencia con respecto al Reglamento 1206/2001, en la medida en que la expresión que emplea el Convenio es "procedimiento ya incoado o futuro", mientras que el instrumento comunitario se refiere a "causa que se prevea incoar".

\subsection{Normas de origen autónomo y delimitación normativa}

El sistema español de Derecho internacional privado (en adelante, DIPr) cuenta con algunas disposiciones relativas a la asistencia internacional y, entre ellas, en particular, el art. 177 de la Ley 1/2000, de 7 de enero, de Enjuiciamiento civil, que, a falta de normas de origen comunitario o convencional, remite a la legislación interna ${ }^{28}$, constituida por los arts. 276-278 de la Ley 6/1985, de 1 de julio, del Poder Judicial (en adelante, LOPJ). En concreto, el citado art. 276 indica que las peticiones de cooperación internacional serán elevadas por conducto del Presidente del TS, del TSJ o de la Audiencia al Ministerio de Justicia, el cual las hará llegar a las Autoridades competentes del Estado extranjero requerido, bien por la vía consular o diplomática o bien directamente, si así lo prevén los Tratados internacionales.

\footnotetext{
${ }^{28}$ Esta disposición señala que: "Cooperación judicial internacional. 1. Los despachos para la práctica de actuaciones judiciales en el extranjero se cursarán conforme a lo establecido en los Tratados internacionales en que España sea parte y, en su defecto, en la legislación interna que resulte aplicable. 2. A lo dispuesto por dichas normas se estará también cuando las autoridades judiciales extranjeras soliciten la cooperación de los juzgados y tribunales españoles".
} 


\section{Doctrina y Jurisprudencia}

Ahora bien, sólo se aplican para el caso de que no lo sea ni el Reglamento 1206/2001 ni el CLH de 1970, tomando en consideración -en todo caso- que la citada norma comunitaria tiene carácter facultativo, de conformidad con la interpretación realizada por el TJCE (véase infra). De otro lado, en cuando a la asistencia judicial pasiva, indica el art. 277 de la LOPJ, que los juzgados y tribunales españoles prestarán a las autoridades judiciales extranjeras la cooperación que les soliciten para el desempeño de su función jurisdiccional, de conformidad con lo establecido en los tratados y convenios internacionales en los que España sea parte y, en su defecto, en razón de reciprocidad según lo previsto en el art. $278^{29}$. Esta disposición prevé las condiciones que ha de reunir la solicitud para que sea prestada la cooperación internacional.

Como disposiciones complementarias, hay que citar el Acuerdo, de 15 de septiembre de 2005, del Pleno del Consejo General del Poder Judicial, por el que se aprueba el Reglamento 1/2005, de los aspectos accesorios a las actuaciones judiciales, en el que, en particular, las secciones $1^{\mathrm{a}}, 2^{\mathrm{a}}$ y $3^{\mathrm{a}}$ (Título IV, Capítulo II) se refieren a las actuaciones judiciales en el extranjero, así como a otras cuestiones relacionadas con la participación de España en la Red de cooperación Judicial Internacional (REJUE) ${ }^{30}$.

Como se sabe, algunos países de la UE son parte del CLH de 1970 sobre obtención de pruebas en el extranjero. Junto con éste, queda abierta la posibilidad de utilizar las vías de cooperación establecidas en la legislación interna de cada Estado miembro de la UE para la realización de pruebas (véase infra) ${ }^{31}$. Prevalece el Reglamento 1206/2001

\footnotetext{
29 La determinación de la existencia de reciprocidad con el Estado requirente corresponderá al Gobierno, a través del Ministerio de Justicia (art. 278, 2응).

${ }^{30}$ Véanse los arts. 74-85 (BOE, de 27 de septiembre de 2005). Este Reglamento sustituye al Acuerdo Reglamentario 5/2003, de 28 de mayo, del Pleno del Consejo General del Poder Judicial, por el que se modifica el Reglamento 5/1995, de 7 de junio de 1995, de los aspectos accesorios de las actuaciones judiciales, en lo relativo a la cooperación jurisdiccional internacional (BOE, de 4 de junio de 2003).

${ }^{31}$ En el caso español, en concreto, quedaría abierta la posibilidad que prevé el art. 77 del citado Acuerdo Reglamentario adoptado por el CGPJ, de desplazamiento al extranjero de Jueces, Magistrados, Secretarios judiciales y funcionarios de la Administración de justicia.
} 


\section{Doctrina y Jurisprudencia}

frente al CLH de 1970 (art. 21), que, no obstante, se aplica cuando la petición de práctica de prueba se dirija o proceda de Dinamarca ${ }^{32}$, así como cuando la solicitud se dirija o provenga de un Estado tercero, parte del mismo. Por lo que respecta a la posición de Reino Unido y de Irlanda, han notificado por escrito su deseo de participar en la adopción y aplicación del Reglamento.

Ahora bien, suscita duda el papel del Reglamento 1206/2001 con respecto, tanto a las normas procesales internas, que regulan la práctica de la prueba, como al Derecho Procesal civil internacional. Cabe indicar que la norma comunitaria no ha sustituido o desplazado tales procedimientos de instrucción existentes en los ordenamientos internos de los Estados miembros de la UE, sino que, por el contrario, ambas reglamentaciones (comunitaria y nacional) se yuxtaponen en el (nuevo) ámbito del proceso internacional, de un $\operatorname{lado}^{33}$.

Y, de otro lado, el propio Reglamento forma parte del Derecho Procesal civil internacional, debiendo emplear el criterio de la primacía para delimitar su aplicación con respecto a otros instrumentos. Sin embargo, como ha indicado el TJCE, en la Sent., de 6 de septiembre de 2012 (As. Lippens y otros), la norma comunitaria tiene carácter facultativo $^{34}$. Por tanto, su aplicación debe ser valorada por la autoridad judicial de cada

\footnotetext{
32 Dinamarca no queda vinculada por los actos jurídicos de desarrollo del Título IV del TCE/TA. Vid. Protocolo núm. 5, anejo al Tratado de Ámsterdam, sobre la posición de Dinamarca.

${ }^{33}$ Vèase, Frigo, M. /Fumagalli, L., “Cap. II. L'assistenza giudiziaria dell'assunzione di prove all'estero”, L'assistenza giudiziaria internazionale in materia civile, Notificazione, Assunzione di prove, Informazioni sul diritto straniero, Cedam, Padova, 2003, pp. 101 y ss.

${ }^{34}$ Ante la demanda de responsabilidad civil presentada en los Países Bajos, se propone el interrogatorio de una de las partes, con domicilio en Bélgica, a la que se cita, de conformidad con el CPC holandés. Ante la negativa a declarar, el Rechtbank de Utrecht (Países Bajos) presentó una cuestión prejudicial al TJCE: el órgano judicial competente de un Estado miembro, que desea interrogar en calidad de testigo a una parte residente en otro, ¿debe aplicar siempre, para llevar a cabo dicho interrogatorio, los procedimientos de obtención de prueba previstos por dicho Reglamento o, por el contrario, tal órgano judicial tiene la facultad de citar ante él a dicha parte e interrogarla con arreglo a su propio Derecho nacional? El Alto tribunal indica que: "el Reglamento 1206/2001, en principio, únicamente es de aplicación en el supuesto en que el órgano jurisdiccional de un Estado miembro decida obtener pruebas con arreglo a uno de los dos procedimientos previstos por dicho Reglamento, en cuyo caso esta obligado a seguir éstos". Véanse las Conclusiones del AG, de 24 de mayo de 2012 (As. C-170/11).
} 
Estado miembro, en función de la oportunidad y eficacia de la obtención de pruebas en el extranjero, con la finalidad de que no se vulnere el derecho a la prueba, si la norma comunitaria limita o no permite la obtención de pruebas de forma más efectiva que las propias normas nacionales.

Esto es, tiene lugar una yuxtaposición entre las regulaciones, comunitaria y nacional (autónoma), en el ámbito del proceso internacional, de un lado y, éstas, a su vez, con la interna, en relación con la regulación de la instrucción ${ }^{35}$.

De otra parte, como es sabido, se planteó esta misma cuestión con respecto al CLH de 1970, acerca de si se trataba de una vía excluyente de las reglamentaciones nacionales sobre obtención de pruebas en el extranjero o, por el contrario, su utilización es facultativa, en el conocido Asunto Société Nationale Industrielle Aérospatiale y Société de construction d'Avions de Tourism versus Corte de Distrito de Estados Unidos para el Distrito Sur de Iowa $(1987)^{36}$.

La Corte Suprema de EEUU consideró que el convenio no tenía preferencia con respecto a las normas federales, sino que se trataba tan sólo de un instrumento, que constituye una alternativa y, por ello, puede ser sustituido por tales reglas federales, si lo

\footnotetext{
35 Por ello, la doctrina considera que se trata de una norma dependiente o subordinada a los ordenamientos procesales nacionales e, incluso, a las reglas de los sistemas de DPCl de cada ordenamiento nacional. Véase, GIOIA, G., “Cooperazione...", loc. cit., pp. 1159 y ss (en relación con el art. 204 del CPC italiano).

${ }^{36}$ Véase texto de la sentencia en, RDIPP, 1987, pp. 829 y ss. Siendo numerosas las referencias bibliográficas sobre el mismo. Véase, entre ellas, HAZARD, G., "Discovery and the rule of the judge in civil law jurisdictions", NDLR, pp. 1022 y ss.
} 


\section{Doctrina y Jurisprudencia}

requiere la justicia del caso ${ }^{37}$. Por último, también presentan interés las relaciones entre organizaciones (p.ej., Conferencia de La Haya y UE), que no pueden ser de exclusión sino de complementariedad ${ }^{38}$. Y, en este marco, es importante analizar las que presentan el Reglamento 1206/2001 y el CLH de 1970, del que forman parte varios Estados comunitarios $^{39}$.

Por lo que se refiere a la materia comprendida en su ámbito de aplicación, el Reglamento prevalece sobre las disposiciones de los acuerdos o convenios bilaterales o multilaterales celebrados por los Estados miembros y, en especial, las del Convenio de La Haya, de 1 de marzo de 1954, relativo al procedimiento civil y del CLH de 1970, entre los Estados miembros que sean parte de dichos convenios (art. 21, $\left.1^{\circ}\right)^{40}$.

\section{BÚSQUeda DE PRUEbas a TRAVÉS DEL PROCEDIMIENTO DE DISCOVERY}

\subsection{Función del procedimiento de discovery}

El incremento de la actividad comercial internacional ha permitido el desarrollo de herramientas y medios destinados a obtener información, que pueda ser utilizada en

\footnotetext{
37 Ibid., p. 1024. Y, en concreto, la posibilidad de utilizar el procedimiento de discovery está directamente relacionada con el derecho a un proceso con todas las garantías, que comprende el principio del due process. Véase, SAROKIN, H.L./ZUCKERMAN, W.E., "Presumed innocent restrictions on criminal discovery in Federal Court belie this presumption”, Rutgers Law Review, 1991, vol. 43, pp. 1089 y ss.

${ }^{38}$ Véase, CRABIT, E., Recherches..., op. cit., p. 524.

${ }^{39}$ Ibid., pp. 500-501.

${ }^{40}$ Ahora bien, el Reglamento no se opone a que dos o más Estados mantengan o celebren acuerdos o convenios entre sí encaminados a facilitar en mayor medida la obtención de pruebas, siempre que sean compatibles con las disposiciones de la norma comunitaria (art. 21, 2ㅇ).
} 
el procedimiento para la prueba de las circunstancias alegadas por las partes, derivadas de la existencia de secretos empresariales, profesionales, así como prohibiciones de intercambiar información.

Así, cada legislador ha previsto cauces distintos para incorporar en el proceso información sobre los hechos relevantes, no siendo sencillo analizar cada uno de ellos, al presentar características muy diferentes: comunicación de información, producción de pruebas, búsqueda de pruebas, exhibición de documentos, son sólo algunos de ellos, que presentan, además, distintas finalidades (conservación de la prueba, aseguramiento del medio de prueba, etc).

El discovery no es -propiamente- una prueba o medio de prueba, sino un procedimiento destinado a la averiguación de los indicios materiales y datos necesarios para preparar un pleito, sin los cuales, el demandante no tendría el material necesario en el sentido de pruebas y verificaciones- para poder plantearlo, de un lado ${ }^{41}$. Y, de otro, no implica el descubrimiento de pruebas (entendida esta expresión en sentido procesal), sino de elementos, documentos o cualquier otro tipo de soporte del que puedan deducirse pruebas en el proceso ${ }^{42}$. Por todo ello, se trata de la búsqueda de indicios

\footnotetext{
${ }^{41}$ El procedimiento de discovery ha sido siempre parte de la pleading in equity (véase, LEVINE, J.B., Discovery in civil procedure: a critical and historical study of the English Law, Thesis, University of Oxford, july 1969 (inédita), p. 25). De otro lado, se ha señalado que es una forma de contrarrestar los inconvenientes del adversary system, que, en ocasiones, se asemeja mucho a una representación teatral. Y, en concreto, el discovery contrarresta el peso de la "own case rule", en virtud de la cual una de las partes puede negarse a proporcionar información a la otra, si no es favorable a los intereses de la primera (véase, LEVINE, J.B., Discovery..., op. cit., p. 7).

${ }^{42}$ El discovery tiene su origen en el Derecho romano y, por tanto, es común a los sistemas jurídicos actuales, que toman su base en tal Derecho. De hecho, las actionem ad exhibendum existentes en los actuales sistemas del Derecho civil proceden también del Derecho romano y puede decirse que constituyen una de las modalidades de acción originarias del actual procedimiento de discovery. Ahora bien, los procedimientos en los sistemas del common law y del civil law se han desarrollado en dos direcciones distintas (lo que no significa que sean opuestas). Así, en los segundos predomina la documentación escrita (prueba documental) y es más significativa la dirección del pleito por una autoridad judicial. En cambio, en los primeros no es tan significativa la aportación de los documentos por escrito y la obtención de pruebas es una actividad de parte. La configuración de los procesos también es distinta, en la medida en que en los sistemas del common law la fase del trial es más breve, de un lado, y de otro, también son más sensibles al hecho de que ha de buscarse la verdad. Sobre la
} 
materiales, más que, propiamente, de la práctica de pruebas, tal y como se prevé en los sistemas pertenecientes al civil law. Se trata de uno de sus principales objetivos.

Ahora bien, el discovery también puede utilizarse para obtener el testimonio de una persona, que no va a poder asistir al $t_{r i a l}{ }^{43}$. Y, en todo caso, la práctica de discovery en Reino Unido se diferencia de la comprensión americana en que, en el primer caso, sólo se permite solicitar el discovery cuando la parte especifica el material que tiene que ser examinado y demuestra la relevancia que puede tener en el caso ${ }^{44}$. Además, es preciso establecer la diferencia entre: "discovery of indirect material" y "proof or direct material", que constituyen los materiales para probar o refutar los hechos objeto del litigio (facts in issue) ${ }^{45}$.

De otro lado, si bien el discovery se sitúa al inicio -antes propiamente- del procedimiento, las diferencias pueden ser significativas entre los distintos sistemas jurídicos, pudiendo producirse situaciones de litispendencia (p.ej.), en la medida en que el discovery se solicite (según lo indicado por cada sistema jurídico) en un momento u otro $^{46}$. Esto es, tras la presentación de la pleading, pero before trial (véase supra) ${ }^{47}$. En

mayor importancia de las facultades de averiguación y obtención de pruebas en el caso de los sistemas de Derecho anglosajón véase, LEVINE, J.B., Discovery..., op. cit. Esta obra proporciona una perspectiva histórica del law of discovery en Inglaterra.

${ }^{43}$ De hecho, en el origen, este procedimiento se empleaba, fundamentalmente, para evitar la pérdida o desaparición de pruebas, en un contexto histórico en el que las distancias entre los justiciables y la administración de justicia eran amplias, de un lado; y, de otro, la separación temporal entre el juicio y el momento en el que tienen lugar los hechos de la causa era muy grande. Véase, RAGLAND, G., Discovery before trial, Callaghan and Company, Chicago, 1932, pp. 13 y ss.

${ }^{44}$ Por este motivo, la delegación británica se opuso a la realización de la discovery en sentido amplio, sugiriendo la reserva del art. 23 del CLH de 1970. Véase, GERBER, D.J., "Extraterritorial Discovery and the Conflict of Procedural Systems: Germany and the United States", AJCL, 1986, vol. 34, pp. 781 y ss.

${ }^{45}$ Véase, ColuINS, L., “The Hague Evidence...”, loc. cit., p. 298.

${ }^{46}$ Así, una de las principales diferencias entre el discovery entre EEUU y Reino Unido reside en que en el primer caso, la mayoría de los procedimientos se realizan con jurado (y tienen carácter oral), de tal modo que, el demandante no va a contar con suficiente información para exponerla en el proceso, si no se le permite averiguar los elementos o datos que le pueden resultar relevantes para su caso con 
todo caso, el discovery es obligatorio (compulsory) y comienza "after pleadings have been eschanged" 48 .

Así, el discovery es anterior al trial (de ahí, que se emplee la expresión pre-trial discovery) $\mathrm{y}$, entre otras funciones, tiene la de evitar sorpresas en el trial cuando el abogado de una de las partes pregunte a la otra por determinados extremos ${ }^{49}$. Este aspecto es importante, porque denota que la forma en la que se desarrolla el proceso ("one day in the court") y la capacidad que tengan las partes de convencer al tribunal ocupan un lugar muy importante en estos sistemas jurídicos ${ }^{50}$. Por ello, es necesario que todos los indicios materiales de prueba estén preparados before-trial. Si no se tienen cuando llegue el trial, el abogado pierde o puede perder el caso ${ }^{51}$. Si se descubren

carácter previo al inicio del procedimiento. Otra diferencia se encuentra en que, en el caso de Reino Unido, el procedimiento de discovery se refiere, principalmente, a documentos y las facultades de la persona que lo solicita están mucho más limitadas, evitando las denominadas "fishing expeditions" (o "fishing proceeeding"), que consisten en el examen de personas, que pueden poner a las partes en el camino para conseguir información (cuando tiene carácter obligatorio), que no se permiten en Reino Unido (véase, ColuıNs, L., "The Hague Evidence...", loc. cit., p. 298). Una tercera diferencia se centra en las personas sobre las que puede realizarse el discovery, dado que en Reino Unido, sólo puede tener lugar con respecto a quien pueda estar implicado en el caso en un ulterior momento, pero no con respecto a terceros. Ahora bien, en cualquier caso, esta limitación está cambiando, pues, como señala la doctrina, han tenido lugar modificaciones en las nuevas reglas de procedimiento tendentes a atribuir mayores facultades de investigación a la autoridad judicial (CROSS). Para un completo análisis de las diferencias entre la regulación del discovery en EEUU y en Inglaterra véase, SIMPSON, R. WM, Civil Discovery and depositions, Trial Practice Library, 2a ed., Wiley Law Publications, New York, 1996.

${ }^{47}$ En todo caso, el discovery se relaciona con la fase pre-trial, al ser el momento adecuado para realizar tales investigaciones o averiguaciones. En el Derecho inglés, el discovery comienza dentro de los 15 días siguientes a la clausura de la fase de alegaciones ("close of pleading").

${ }^{48}$ Véase, CAMPBELL, D, Serving..., op. cit., 1998, p. 458.

${ }^{49}$ Si las respuestas no vienen bien a la parte a quien representa, puede quedar mal ante los ojos del tribunal y del jurado. Véase, LeVINE, J.B., Discovery..., op. cit., pp. 6-7.

50 Es importante también otro aspecto, que señala la doctrina, relativo a la mayor importancia que ha tenido tradicionalmente el procedimiento en el Derecho inglés (ibid.).

51 Esto es, si el demandante no logra probar lo que solicita, pierde el caso. Esto es, si no existen suficientes pruebas para demostrar lo que alega, no se sobresee el asunto sino que se pierde. Véase, MuRPhy, P., Evidence and Advocacy, 5a ed., 1998, pp. 1-23. 


\section{Doctrina y Jurisprudencia}

nuevos elementos (pruebas, acontecimientos, etc.), le pueden hacer perder el caso ante los ojos del tribunal ${ }^{52}$.

De otra parte, cabe apreciar la diferencia entre la petición de discovery y de "produce some documents", pues en el primer caso, se trata de documentos que ya tiene el demandado, mientras que en el segundo se trata de que el demandante (plaintiff) estuviera autorizado a pedir testimonio al demandado (produciendo, así, elementos de prueba, de ahí que reciba el nombre de "producir", esto es, "production of documents") ${ }^{53}$. También cabe decir que en los distintos idiomas y, en concreto, en lengua inglesa, existen distintos términos para aludir a ideas distintas: gathering evidence y discovery ${ }^{54}$.

Por tanto, una de las principales diferencias entre la discovery y otros medios de instrucción (confesión, etc.) consiste en que el primero es un auténtico procedimiento de investigación, mediante el cual no se trata sólo de obtener pruebas, sino todo tipo de información a través de la cual hacer averiguaciones posteriores ${ }^{55}$. Al mismo tiempo, es posible que sea más amplia la posibilidad de obtener todo tipo de indicios materiales en la fase de discovery, que durante el trial, lo que puede dar lugar a que determinados

\footnotetext{
${ }^{52}$ En este sentido, en el Derecho inglés se considera que "una parte no puede ser sorprendida en el proceso (trial)". Véase, CAMPBELL, D., Serving..., op. cit., p. 459.

${ }^{53}$ Véase, LEVINE, J.B., Discovery..., op. cit., pp. 44-48.

${ }^{54}$ Véase, Coluns, L., “The Hague Evidence...”, loc. cit., p. 338.

${ }^{55}$ Las órdenes de disclosure también revisten la forma de injunctions. El desarrollo que han tenido estas decisiones en el ámbito comercial desde la década de los 70 del pasado siglo hasta la actualidad, ha hecho que se haya planteado la cuestión de su validez extraterritorial, relacionada, a su vez, con la competencia internacional de la autoridad que las pronuncia. Véase, MCLACHLAN, C., "The jurisdictional limits of disclosure orders in transnational fraud litigation", ICLQ, 1998, vol. 47, pp. 3 y ss; WALKER, G., "Bank Remedies: Judicial and Arbitral Recovey", N. HORN/J.J. NORTON, Non-Judicial Dispute Setttlement in International Financial Transactions, vol. 13, Kluwer, London, 2000, p. 41.
} 
indicios materiales recabados durante el discovery no puedan servir de prueba (proof) en el trial $^{56}$.

Por último, Inglaterra e Irlanda son dos de los países del common law, que tienen más desarrollada la discovery. Es decir, que atribuyen mayores poderes para ello ${ }^{57}$. En todo caso, pudiendo apreciarse diferencias entre la regulación de este procedimiento en los países del common law, interesa centrarse a continuación en si, realmente, existen inconvenientes para aceptar su práctica en el espacio europeo (véase punto 3), de un lado, y, de otro, si se trata es desconocido en los sistemas del civil law ${ }^{58}$.

\section{2.¿Existe el discovery en los sistemas del civil law?}

No puede decirse que el procedimiento de discovery exista sólo en los países pertenecientes a la órbita del common law en el momento actual ${ }^{59}$. Por el contrario, las reformas que han tenido lugar durante las últimas décadas en los países continentales de la UE ponen de relieve que se han ampliado las facultades de investigación de los

\footnotetext{
${ }^{56}$ En todo caso, el procedimiento de discovery no constituye tan sólo un conjunto de reglas que lo regulan, sino que recibe un tratamiento científico y legislativo individualizado, tratándose, el Law of Discovery, de un ámbito independiente -desde ambos planos- del Law of Evidence, con el que no debe confundirse. Véase, en particular, SIMPSON, R. WM., Civil Discovery and depositions, Trial Practice Library, 2a ed., Wiley Law Publications, New York, 1996.

${ }^{57}$ Véase, CAHILL, E., Discovery in Ireland, Sweet and Maxwell, Dublin, 1996.

${ }^{58}$ Para un extenso tratamiento de las divergencias y similitudes entre la práctica del discovery en EEUU y en Reino Unido véase, LeVINE, J.B., Discovery. A Comparison between English and American Civil Discovery Law with Reform Proposals, Clarendon Press, Oxford, 1982; CLARK, D.S., "Chapter 16. Civil Procedure", Introduction to the law of the United States, Edited by D.S. Clark and T. Ansay, Kluwer, 2002, pp. 374 y ss. Este último autor señala que puede resultar chocante que el sistema de EEUU sea más parecido al modo germánico de las tribus, que el actualmente existente en Alemania (documental y escrito).

${ }^{59}$ Para una visión del procedimiento de discovery y su regulación en los países de la UE véase, TAYLOR, T./COOPER, N., European Litigation Handbook, Sweet and Maxwell, London, 1995, pp. 47 y ss (capítulo 4).
} 
hechos por la autoridad judicial y, en ocasiones, por las partes, en función de la concreta concepción del Derecho de la prueba y de la actividad de instrucción existente en cada uno de ellos ${ }^{60}$.

En todo caso, las reformas que han tenido lugar en los ordenamientos procesales de los países miembros de la UE han ampliado los deberes de las partes en relación con la impartición de la justicia, de forma que se han incorporado distinto tipo de obligaciones $\mathrm{y}$, entre ellas, en particular, de exhibición y comunicación de documentos ${ }^{61}$. De otro lado, también se pone de relieve la concepción individualista existente en los ordenamientos francés e inglés, en virtud de la cual, las partes pueden decidir qué tipo de pruebas aportan al proceso ${ }^{62}$.

Por ello, se considera que es -verdaderamente- difícil considerar que el procedimiento civil francés tienda a buscar la verdad en la actualidad, sino -más bien- a asegurar una competencia leal entre las partes, de tal manera que no puedan ser libres de ocultar la existencia de un documento, que sería útil al adversario ${ }^{63}$. En concreto, en el

\footnotetext{
${ }^{60}$ Véase, HAZARD, G.C., "Discovery and the rule of the judge in civil law jurisdictions", NDLR, pp. 1019 y ss.

61 En el Derecho español, la exhibición de documentos es una diligencia preliminar, que permite al particular solicitar a la autoridad judicial que quien tenga la cosa, la exhiba; que quien tenga los documentos y cuentas de la sociedad, los enseñe, entre otras (art. 257 LEC/2000). Ahora bien, también pueden solicitarse diligencias de obtención de datos, en particular, en el ámbito de la propiedad intelectual e industrial, tras la entrada en vigor de la Ley 19/2006. Sin embargo, las diligencias preliminares también pueden consistir en la práctica de una declaración y no sólo en la exhibición. En el Derecho francés, la exhibición de documentos está comprendida en la obligación de production de pièces (art. $11 \mathrm{NCPC}$ ), que permite a la autoridad judicial ordenar una injonction, cuyo alcance extraterritorial se discute.

${ }^{62}$ Véase, Jolowicz, J. A., "La production forcée... », loc. cit., p. 170.

63 Se indica, por ello, que las diferencias en el modo de presentar las pruebas en los sistemas del common law y del civil law no son tan nítidas si se analiza con detenimiento (ibid., p. 172). De hecho, podría ser más importante la posibilidad de obtener pruebas de conformidad con el NCPC francés, que con el procedimiento de discovery. Véase, PAULSSON, J., "Overview of Methods of Presentig Evidence in Different legal systems » en, A.J. van den Berg (ed.), Planning Efficient Arbitration Proceedings/The Law Applicable in International Arbitration, Kluwer, La Haya, 1999.
} 
Derecho francés, la Ley núm. 72-625, de 5 de julio de 1972, introdujo en el Code Civil una nueva disposición, que regula la production de pièces (obtention des piéces) ${ }^{64}$. El art. 10 del Code Civil señala que: “chacun est tenue d'apporter son concours á la justice en vue de la manifestation de la vérité».

El art. 11, 2 del Nuevo Código de Procedimiento civil francés (NCPC) formula el principio (production forcée d'une preuve) y los arts. 138-142 regulan su régimen ${ }^{65}$. Por ello, uno de los principales cambios que tuvo lugar tras la citada reforma fue la introducción de un poder de injonction para la producción de documentos (art. 11, 3º), bajo la pena de astreinte, a cargo de quien los tenga (parte o terceros) ${ }^{66}$. Esto es, se atribuye competencia a las autoridades judiciales francesas para ordenar la práctica de medidas de instrucción (de prueba) incluso antes del inicio del proceso de fondo, bien a través del procedimiento contradictorio del référé, bien sur requête (que no es contradictorio) ${ }^{67}$.

Por tanto, pueden adoptarse medidas destinadas a obtener pruebas antes del inicio del proceso en el caso del Derecho francés, al igual que en el anglosajón en la fase pre-

\footnotetext{
${ }^{64}$ Véase, MARRAUD, C., "Le droit á la preuve. La production forcée de preuves en justice. Décrets núm. 71-740, du 9 sept. 1971, núm. 72-684, du 20 juillet 1972 et num. 72-788 du 28 août 1972)", JCP, 1973-I (doc. 2572); JolowiCz, J. A., "La production forcée des pièces. Droit français et anglais", Nouveaux juges, nouveaux pouvoirs?, Mélanges en l'honneur de Roger Perrot, Dalloz, Paris, 1996, pp. 167 y ss.

65 Incluso el árbitro puede ordenar a las partes la producción de elementos de prueba (art. 1460, 3ạ NCPC). Véase, con carácter general, BRAHIC-LAMBREY, C., L'injonction. Étude de la dynamique d'un processus, tomos I y II, Press Univ. d'Aix Marseille, 2003.

${ }^{66}$ Véase, CORNU, G., "Les principes directeurs du procès civil par eux-mêmes (fragments d'un état des questions)" en, Études offertes á Pierre Bellet, Litec, Paris, 1991, pp. 83 y ss. Y, de otro lado, este autor explica cuáles han sido las principales líneas de la reforma.

${ }^{67}$ En contra de la tesis de S. Clavel, otro sector de la doctrina señala que el juge des référés puede ordenar también la producción obligatoria de documentos, como indica la jurisprudencia. Véase, HORY, A., "Mesures...", loc. cit., p. 217.
} 


\section{Doctrina y Jurisprudencia}

trial. Ahora bien, hay que plantear la pregunta de si la noción de "medidas de instrucción" es idéntica a discovery. Sin embargo, incluso ampliándose las facultades de la autoridad judicial en el ámbito del descubrimiento de indicios materiales de prueba, aún pueden apreciarse diferencias con respecto al procedimiento de discovery, porque una diferencia principal entre los países de Derecho común y los pertenecientes al Derecho civil es que éstos consideran que la búsqueda de pruebas es un acto de autoridad y no una tarea de las partes ${ }^{68}$.

Esta perspectiva revela la diferente concepción existente entre estos dos sistemas en relación, no sólo con la prueba en un proceso, sino también con el papel que corresponde a las partes con respecto a su desarrollo o desenvolvimiento. Y, en todo caso, no es lo mismo, presentar pruebas (proposición de prueba), obtener o practicar la prueba, que descubrir (discovery) indicios materiales, dado que en este último caso, es preciso indagar su propia existencia ${ }^{69}$.

Si la prueba tiene una finalidad de acreditar la veracidad de los hechos que se aportan en el proceso, no sería admisible utilizar la fase de prueba para averiguar elementos que puedan servir para aportar otras pruebas, que no se han presentado, pero

\footnotetext{
68 Véase, MAIER, H.G., «Extraterritorial discovery: cooperation, coercion and the Hague Evidence Convention», Vanderbilt Journal of Transnational Law, 1986, vol. 19, núm. 2, pp. 239 y ss; y VAN SIMPSON, R, Civil Discovery..., op. cit., p. 495 (nota 52).

69 Así, el TS alemán ha establecido en Sent. de 12 de julio de 1984 que: "darf eine Parteie beweiserhebliche Tatsachen nicht erst durch die Beweisaufnahme zu erfharen suchen, um si dann zur Grundlage ihres Prozessvortrages zu machen" ("no pueden utilizarse los procedimientos de prueba para demostrar hechos que constituyan el fundamento de la demanda", traducción propia). Véase texto en, Juristenzeitung, 1985, p. 183 (comentada por R. Sürner). Véase, sobre este particular, SCHÜTZE, R.A., "Die Annerkennung und Vollstreckung US-amerikanischer Zivilurteile, die nach einer pre-trial-discovery ergangen sind, in der Bundesrepublik Deutschland", Festschrift für Ernst C. Stiefel, München, 1987, pp. 697 y ss. De otro lado, como es sabido, los abusos a los que puede llevar tal investigación acerca de pruebas condujeron a la delegación inglesa a proponer una reserva al CLH de 1970. Dicha reserva tuvo la finalidad de evitar la realización de pruebas de forma fishing, esto es, sin el respeto de los derechos y garantías fundamentales del individuo, no sólo relacionadas con el procedimiento, sino las más generales.
} 


\section{Doctrina y Jurisprudencia}

que pueden aportarse, si se obtienen a través de tales actos de averiguación o investigación $^{70}$. Así, la principal diferencia entre las normas del Derecho procesal de los sistemas del common law y del civil law reside, no sólo en la diferente amplitud de la fase llamada pre-trial, sino también en las distintas facultades que corresponden tanto a las partes como a la autoridad judicial en el discovery (indagación o descubrimiento) de los indicios materiales de prueba ${ }^{71}$.

Y, en este sentido, el discovery no constituye una medida o acto de instrucción, sino que tiene que ver con disclosure, que significa, a su vez, sacar a la luz, desvelar información, elementos, datos, etc ${ }^{72}$. Por ello, no puede confundirse obtención (ni producción) de pruebas con discovery, porque este último conlleva la disclosure de datos, elementos, etc. que no se conocían hasta el momento en que comenzó la investigación de los hechos.

Ahora bien, con respecto a esta cuestión, todos los ordenamientos pertenecientes al civil law presentan las mismas características -extremo que destacan de manera importante y principal los autores que se ocupan del Derecho procesal comparado-, ni

\footnotetext{
${ }^{70}$ Otra de las principales ventajas del discovery consiste en que no dilata la marcha del proceso, sino que tiene como principal característica la rapidez o celeridad, porque no se ha de esperar a la citación judicial (subpoena duces tecum) para que tenga lugar la declaración de un testigo, p.ej. (véase, LEVINE, J.B., Discovery..., op. cit., p. 6).

${ }^{71}$ De hecho, una de las principales dudas que suscitaba la reforma del art. 142 del ZPO alemán, que entró en vigor el 1 de enero de 2001 (Bundesgesetzblatt, 2001, l, 40, pp. 1887 y ss.), fue el temor a que se introdujese una ampliación excesiva de los poderes del juez, parecida al procedimiento americano de discovery of documents. Véase, DI FAZZIO, G., "La riforma dell'esibizione di documenti nel processo civile tedesco", RTDPC, 2006, p. 154.

72 Cabe apreciar la importancia de esta cuestión en el ámbito comercial, en el campo del Derecho antitrust, así como en los litigios relacionados con la empresa. La American Bar Association (sección antitrust Law) publicó un libro sobre Obtaining Evidence Abroad, que tiene sumo interés, porque se refiere a la importancia de este tipo de procedimientos de prueba cuando se trata de litigios en el ámbito comercial, especialmente, internacional. $Y$, en este sentido, es conocido el "Asunto Aèrospatiale", resuelto por el Tribunal Supremo americano, por Sentencia, de 15 de junio de 1987 (RDIPP, 1987, pp. 827 y ss). Para un amplio comentario del mismo véase, entre otros, TROCKER, N. , "II contenzioso...", loc. cit., pp. 622 y ss.
} 


\section{Doctrina y Jurisprudencia}

tampoco los del common law, y, al mismo tiempo, pueden existir coincidencias entre ambos sistemas en algunos aspectos ${ }^{73}$. De otro lado, no hay necesidad de discovery en los sistemas de civil law, al tratarse de procedimientos -fundamentalmente- escritos, en los que falta la oralidad y, por ello, no hay ninguna ventaja táctica o estratégica a la que haya que adelantarse ni debe sacarse partido (del aspecto) de la sorpresa ${ }^{74}$.

De otro lado, si bien puede existir una fase parecida a la del pre-trial (que, en cualquier caso, comienza con las alegaciones o pleading) en los sistemas del civil law, en éstos los poderes de investigación de las partes son mínimos, si se comparan con los que les corresponden en los sistemas del common law. Y la explicación acerca de esta mayor atribución a las partes de facultades de indagación y búsqueda de indicios materiales en la concepción del common law puede estar relacionada con el hecho de que el juez cuenta con menores posibilidades de dirigir el proceso, al menos, en esta fase de instrucción ${ }^{75}$.

No obstante, la doctrina señala que, si bien tanto en el Derecho francés como en el alemán existen mecanismos y expedientes parecidos o que cumplen la misma función que el pre-trial discovery en EEUU, las técnicas que existen en estos sistemas jurídicos para averiguar la verdad (descubrir indicios materiales de prueba) no son comparables

\footnotetext{
${ }^{73}$ Puede apreciarse este parecido en el caso de la etapa de la audiencia previa, que regula el art. 414 de la LEC/2000 española, que permite realizar algunos de los actos que se desarrollan en la fase pre-tria del procedimiento de los sistemas del common law: identificación de las cuestiones que alegan las partes, recolección de pruebas, conciliación, entre otras.

${ }^{74}$ La doctrina señala que: "discovery is less necessary because there is little, if any, tactical or strategic advantage to be gained from the element of surprise" (véase, MERRYMAN, J.H., The civil law..., op. cit., p. 113). Esto es, en el common law es preciso evitar la sorpresa, porque, al tratarse de un procedimiento concentrado, el abogado no puede permitirse el lujo de que sean descubiertas o presentadas pruebas nuevas en la fase del trial.

75 En la doctrina, G. HAZARD aborda las diferencias en cuanto al papel que tienen juez y partes en la presentación de las pruebas (véase, "Discovery and the rule of the judge in civil law jurisdictions", NDLR, 1993, pp. 1019 y ss). Véase, también, sobre este particular; GLASSER, C., "Civil procedure and lawyers. The adversary System and the Decline of the orality Principle", MLR, 1993, pp. 307-324.
} 


\section{Doctrina y Jurisprudencia}

con las que prevé el Derecho americano ${ }^{76}$. De otro lado, y en este mismo sentido, se han puesto de relieve las ventajas que pueden presentar para las empresas las reglas que regulan el procedimiento de discovery en EEUU, pudiendo tener lugar, incluso, un "discovery shopping",

En el Derecho alemán, si bien la aportación de los elementos de prueba no incumbe al juez, que no ha de indagarlos de oficio, de conformidad con el principio dispositivo (Verhandlungsmaxime), desde el año 1933 se han introducido en el Código de procedimiento civil de 1877 (Zivilprocessordnung) una serie de disposiciones, que indican que las partes deben hacer declaraciones completas y conformes a la verdad sobre los hechos del proceso $^{78}$. De otra parte, tuvo lugar una reforma importante en 2002, para permitir la exhibición de documentos, mediante un proceso autónomo (art. $142 \mathrm{ZPO})^{79}$.

Se ha señalado, sin embargo, que no puede establecerse una equivalencia de este procedimiento con el discovery anglosajón, en la medida en que no tiene carácter exploratorio $^{80}$. En el caso de Austria, el discovery sólo se prevé para que cada una de las partes pueda aportar los indicios materiales que considere relevantes para la defensa de

\footnotetext{
76 Véase, von Mehren, A./ Rusell Gordley, J., The civil law System, Little, Brown and Co., Boston and Toronto, 1977, p. 1158.

${ }^{77}$ El art. 1782 del Código judicial de USA permite que todo tribunal americano pueda arbitrar todos los procedimientos o técnicas de instrucción existentes en el Derecho procesal americano a petición de una autoridad judicial extranjera y, entre ellos, la discovery. Véase, LEGUM, B., «Discovery in aid of foreign proceedings provided by United States Courts», RDAl, 1998, núm. 7, pp. 747 y ss.

${ }^{78}$ Sin embargo, se considera que no se parece a la discovery..Véase, NADELMAN, K.H., «De la preuve en Droit allemand», Bulletin de la Société de législation comparé, vol. 68, 1939, pp. 173 y ss.

${ }^{79}$ El nuevo art. 142 del ZPO configura la exhibición de documentos como un deber procesal. Véase, DI FAZZIO, G., "La riforma dell'esibizione di documenti nel processo civile tedesco", RTDPC, 2006, pp. 143 y ss.

${ }^{80}$ Ibid., p. 154.
} 


\section{Doctrina y Jurisprudencia}

sus propios argumentos. En el Derecho belga tampoco existe un procedimiento de discovery comparable con el que se conoce en Reino Unido.

Por último, el Derecho italiano regula la posibilidad de "produrre la prova" en el art. 184 del $\mathrm{CPC}^{81}$. Se han planteado una pluralidad de reformas, siguiendo las que han tenido lugar en Francia (en donde se ha introducido el procedimiento de rèfèrè), propuestas por la Comisión Vaccarela ${ }^{82}$. Dichas modificaciones se relacionan con el procedimiento sumario (provvedimento sommario) en el ámbito societario, que permite a las partes (a sus abogados) desarrollar una articulada actividad de instrucción prejudicial (istruttoria pre-giuduziale) de cara al futuro proceso ${ }^{83}$.

\section{PRÁCTICA DEL DISCOVERY EN EL REGLAMENTO 1206/2001 Y EN EL CONVENIO DE LA HAYA DE 1970}

\subsection{El controvertido art. 23 del Convenio de La Haya de 1970}

A) Génesis de la reserva

El tratamiento de la práctica de discovery en el extranjero en el momento actual en el espacio judicial europeo se relaciona, sin duda, con la regulación dada a esta cuestión por el CLH de 1970, anterior al Reglamento $1206 / 2001^{84}$. Los abusos a los que

\footnotetext{
${ }^{81}$ Véase, Comoglio, L. P., “Istance istruttorie e poteri del giudice ex art. 184 CPC”, Riv. Dir. Proc., 1999, pp. 989 y ss.

82 Véase, entre otros, RICHI, E. F., "Verso un nuovo processo civile?", Riv. Dir. Proc., 2003, pp. 211 y ss; CeCChella, C., "Il rèfèrè italiano nella riforma della società", Riv. Dir. Proc., 2003, pp. 1130 y ss.

${ }^{83}$ Véase, RICHI, E.F., "Verso un nuovo...", loc. cit., pp. 221-223.

${ }^{84}$ Como se ha señalado, el discovery no es una mera prueba, sino un procedimiento destinado, fundamentalmente, a obtener cualquier información, que pueda ser de utilidad para la parte para preparar el proceso (trial). Véase, SIMPSON, R. WM., Civil Discovery and depositions, Trial Practice Library, 2a ed., Wiley Law Publications, New York, 1996, p. 5.
} 
conducía la investigación acerca de la propia existencia de indicios materiales para demostrar los hechos que alegan las partes condujeron a la Delegación inglesa a proponer - a última hora de la negociación del convenio- la posibilidad de realizar una reserva en el art. 23 del CLH de 1970, según el cual: "todo Estado contratante podrá declarar en el momento de la firma, ratificación o adhesión, que no ejecutará las comisiones rogatorias que tengan por objeto el procedimiento conocido en los países del common law con el nombre de pre-trial discovery of documents" ${ }^{85}$.

Las principales dudas que ha suscitado su aplicación se refieren a su propia génesis, así como a su diversa interpretación desde las órbitas americana y europea, pudiendo decirse que se ha tratado de un artículo muy controvertido, que ha frustrado -hasta cierta medida- las expectativas de los Estados parte ${ }^{86}$. En principio, dicha reserva tuvo la finalidad de evitar tener que ejecutar órdenes extranjeras de discovery, que tuviesen la finalidad de obtener la información en la fase pre-trial del procedimiento, o que se tratase de información que no estuviese indicada en la orden ${ }^{87}$.

\footnotetext{
${ }^{85} \mathrm{Y}$, en concreto, el Gobierno español realizó una reserva en el siguiente sentido: "España no acepta las comisiones rogatorias derivadas del procedimiento pre-trial discovery of documents conocido en los países del common law".

${ }^{86}$ Así, el sentido de esta disposición puede ser distinto según se analice desde la óptica de EEUU o europea, en función de la diversa concepción sobre el proceso existente en cada ordenamiento. Tratan este aspecto, ColuINS, L., "The Hague Evidence...", loc. cit., pp. 289 y ss; y GERBER, D.J., «Extraterritorial Discovery and the Conflict of Procedural Systems: Germany and the United States", AJCL, 1986, vol. 34, pp. 781 y ss.

${ }^{87}$ La doctrina señala que dicha reserva tuvo la finalidad de que pudieran limitarse las citadas órdenes de discovery para proporcionar información documental en la fase anterior al proceso, así como para evitar que se ejecutasen decisiones de discovery que tuviesen un carácter general (véase, NAISH, J./THOMAS, R., "United Kingdom", The Comparative Law Yearbook of International Business, Special Issue, 1998, p. 462).
} 


\section{Doctrina y Jurisprudencia}

Esto es, dicha reserva tuvo la finalidad de limitar las peticiones de discovery procedentes de EEUU, parte también del CLH de 1970, precisamente, por la distinta concepción existente en cada uno de estos ordenamientos ${ }^{88}$. De otro lado, se trataba de evitar la realización de pruebas de forma fishing (fishing expeditions) esto es, sin el respeto de los derechos y garantías fundamentales del individuo, no sólo relacionadas con el procedimiento, sino también las más generales ${ }^{89}$.

B) Sentido del art. 23 del Convenio de La Haya

La Sentencia de la House of Lord en el Asunto Westinghouse ("In re Westinghouse Electronic Corporation Uranium Contract Litigation") pronunciada en 1978, ilustra las dudas que se presentaron con ocasión de las reuniones de la Comisión especial encargada de supervisar la aplicación del Convenio ${ }^{90}$. En todo caso, la respuesta del Alto Tribunal británico a la petición de comisión rogatoria americana es que no era procedente su ejecución $^{91}$.

${ }^{88}$ Es ilustrativo de esta explicación el Asunto Westinghouse Electronic Corp., para cuya resolución se tuvo en cuenta el hecho de que la comisión rogatoria americana solicitara la práctica del discovery. Véase, "Obtaining evidence for proceedings in other jurisdictions», CJQ, vol. 21, 2002, pp. 83 y ss.

${ }^{89}$ Véase, EDWARD, D.M., "Taking of Evidence abroad in civil or commercial matters", ICLQ, 1969, vol. 18, p. 650 .

${ }^{90}$ La decisión está publicada en $W L R, 1978$, vol. 81 y recogida también en, International Legal Materials, 1978, vol. 38. Se trató de un tema controvertido, en el que se mezclaron varios aspectos, entre ellos, el hecho de que se tratase de una comisión rogatoria destinada a desplegar efectos en un procedimiento criminal (Defensa de la competencia), pero también se trataron otras cuestiones. Véase, Report on the work of the special commission on the operation of the Convention of 18 march 1970 on the taking of evidence abroad in civil and commercial matters, June 12-15, 1978 (prepared by Permanent Bureau), International Legal Materials, 1978, vol. 17, pp. 1425 y ss.

${ }^{91}$ En concreto, el Alto Tribunal británico señala que: "pre-trial discovery is distinct from the obtaining of evidence for the trial and that the approach of this court must be to allow letters rogatory only in so far as they are confined to obtaining evidence and are not requiring the exercise of pre-trial discovery". Texto reproducido por, ColuINs, L., "The Hague Evidence...", loc. cit., p. 307. 


\section{Doctrina y Jurisprudencia}

Ahora bien, el hecho de que el Estado requerido haya realizado la citada reserva prevista en el art. 23 del CLH de 1970 no implica necesariamente que no pueda solicitarse -a través del Convenio- la obtención de pruebas, que tengan lugar antes, incluso, del inicio del procedimiento de fondo. Por el contrario, el propio texto del CLH de 1970 indica con claridad, que la práctica de diligencias de obtención de pruebas se empleará para aportar el medio de prueba obtenido en el extranjero al procedimiento que ya se encuentre incoado o que se incoe en un futuro en el Estado requirente.

Esta referencia es interesante para analizar qué aspectos quedaron excluidos, en realidad, del pre-trial discovery of documents, a propuesta de la delegación británica, lo que no quiere decir que se haya excluido en todo caso, ni tan siquiera para los Estados que hayan realizado la reserva ${ }^{92}$. Por el contrario, el Informe de la Comisión especial sobre el funcionamiento del CLH de 1970 (preparado por la Oficina Permanente, en su reunión de junio de 1978) explica que el sentido de la reserva tiene que limitarse a aquellos casos en los que la comisión rogatoria carezca de la suficiente explicación, de tal forma que no permita identificar los documentos que tienen que ser obtenidos o examinados ${ }^{93}$.

De otro lado, la cuestión de si el discovery es excesivo no deriva de su funcionamiento (dado que existen casos en los que debe permitirse para poder esclarecer los hechos), sino del abuso en el ejercicio de la competencia ${ }^{94}$. Por tanto, se

\footnotetext{
92 Véase, SCHORE, L./SMITH, H., "Making applicants take evidence properly: challenges to letters of request", ICL, 1998, pp. 41 y ss.

93 Véase, Report on the work of the special commission on the operation of the Convention of 18 march 1970 on the taking of evidence abroad in civil and commercial matters, June 12-15, 1978 (prepared by Permanent Bureau), International Legal Materials, 1978, vol. 17, p. 1428.

${ }^{94}$ Si se utiliza un criterio personal exorbitante (como el forum presentiae, esto es, el sólo hecho de que el demandado se encuentre en el territorio de un concreto Estado, justifica la competencia internacional de sus autoridades), que va a acompañado del ejercicio de jurisdicción de ejecución con respecto a la realización de pruebas, entonces, sí podría considerarse que no debe admitirse el discovery (As. Westiinghouse).Véase, ColuINS, L., “The Hague Evidence...”, loc. cit., p. 311.
} 


\section{Doctrina y Jurisprudencia}

podría poner el límite de que el discovery no podría realizarse en el extranjero, si la competencia de la autoridad del Estado requirente es exorbitante, cuando el caso tiene mínimos contactos con el foro.

\subsection{Tratamiento del discovery en el Reglamento 1206/2001}

No se ha recogido en el texto del Reglamento 1206/2001 una referencia específica a este procedimiento, que transcurre usualmente en una etapa previa o anterior al proceso, que es conocida como pre-trial ${ }^{95}$. Un sector doctrinal considera que el discovery está incluido en el Reglamento 1206/2001 y que el hecho de que no se haya recogido una mención expresa ha de interpretarse en el sentido de su falta de utilidad, habida cuenta de que EEUU no es un Estado comunitario y que las principales dificultades que se habían presentado con el discovery se derivaron de las diferencias existentes en su concepción entre los Estados de la UE y, en particular, Reino Unido, de un lado; y EEUU, de otro ${ }^{96}$.

De forma algo más matizada, y realizado una interpretación de la entera norma comunitaria, se considera que no comprende las actividades de instrucción, que tenga

\footnotetext{
${ }^{95}$ Considera la doctrina que de las Declaraciones del Consejo para la adopción del Reglamento del 15 y 17 de mayo de 2001 (textos 8633/01, de 15 de mayo de 2001, p. 42; y 8928/01, de 17 de mayo de 2001, p. 46), así como del Documento del Grupo de Trabajo del art. 29 sobre protección de datos [Documento de trabajo 1/2009 (WP 158), de 11 de febrero del Grupo de Trabajo del art. 29 sobre protección de datos] se excluye esta figura y su compatibilidad con el Derecho procesal civil europeo (véase, ELVIRA BENAYAS, Ma J.,"Recensión a la monografía G. Esteban de la Rosa, La prueba en el espacio europeo de justicia, Editorial Académica Española, Berlín, 2012”, REEl, núm. 25, junio de 2013. Ahora bien, ésta no es la interpretación que realiza la Abogada General (Sr. J. Kokott) en sus conclusiones presentadas en el As.Tedesco (http://eur-lex.europa.eu/LexUriServ/LexUriServ.do?uri=CELEX:62006CC0175:ES:PDF). En sentido favorable a la inclusión del discovery en el Reglamento, VILLAMARÍn LÓPEZ, M.L., La obtención de pruebas en el proceso civil en España. Estudio del Reglamento 1206/2001, de 28 de mayo, Colex, Madrid, 2005, p. 61, quien considera que las citadas Declaraciones tienen sólo valor interpretativo.

96 Véase, LeBEAU, D./NiBOyET, M.L., “Regards croisés du processualiste et de l'internationaliste sur le Règlement CE, du 28 mai 2001, relatif á l'obtention des preuves civiles á l'étranger", Gaz. Pal., enerofebrero 2003, p. 225.
} 


\section{Doctrina y Jurisprudencia}

una finalidad distinta a la probatoria, esto es, exploratoria o de búsqueda de fuentes de prueba $^{97}$. Y, en este sentido, se ha pronunciado también la Abogada General, la Sra. J. Kokott, en las conclusiones presentadas, el 18 de julio de 2007, en el As. Tedesco, sobre el que no llegó a pronunciarse el TJCE, al haberse puesto término al procedimiento que dio origen a la cuestión prejudicial (Auto, de 27 de septiembre de 2007).

En concreto, indica que no se justifica la denegación (por la autoridad inglesa) de la práctica de la prueba solicitada por el Tribunale civile di Genova, que consideró que la descripción solicitada no está comprendida en el ámbito del Reglamento 1206/2001. Por el contrario, de conformidad con su art. $1,2^{\circ}$, hay que diferenciar dos situaciones: de un lado, ha de declararse la inadmisibilidad de un exhorto de obtención de pruebas cuando los documentos, cuya presentación se solicita, conducen a la localización de elementos de prueba susceptibles de ser utilizados, pero no están destinados en sí mismos a fines probatorios en el marco del proceso (los denominados "train of enquiry", búsqueda ilícita de material probatorio relevante $)^{98}$.

Y, de otro, un exhorto de obtención de pruebas dirigido a la presentación de documentos, que son encontrados una vez ejecutado el exhorto, es admisible si los documentos son designados con suficiente precisión y guardan una relación directa con el objeto litigioso. Por último, existen otras vías para impedir que se ejecute una petición de discovery en el extranjero, mediante la denegación del reconocimiento de la decisión extranjera, si la utilización del procedimiento de discovery ha sido abusiva.

\footnotetext{
97 Por ello, no comprende: las investigaciones procesales, que estén privadas de un objeto claramente especificado; las indagaciones de prueba, que no tengan una previa definición del thema provandum; la actividad de indagación que, antes que al control probatorio de una situación de hecho ya definida y a la determinación de la posibilidad de vincularle consecuencias jurídicas, sirva para encontrar hechos o informaciones relevantes para definir el objeto de la contienda (véase, Trocker, N. , "Note sul Regolamento...", loc. cit., p. 680).

${ }^{98}$ Conclusiones de la Abogada General, supra citadas, marg. 72.
} 
La existencia del procedimiento de discovery en alguno de los ordenamientos de los Estados miembros de la UE sigue suscitando la duda acerca de si es posible utilizar el Reglamento 1206/2001 para solicitar la cooperación de las autoridades judiciales comunitarias para la obtención de de indicios o material de prueba. Si bien la norma no comprende las indagaciones de pruebas que no tengan una previa definición del thema provandum; debe admitirse un exhorto de obtención de pruebas dirigido a la presentación de documentos, si se designan con suficiente precisión y guardan una relación directa con el objeto del litigio.

Como apuntó la Abogada General en las conclusiones de 18 de julio de 2007, presentadas en el As. Tedesco (supra referidas), los elementos de prueba han de estar determinados de forma tan precisa que se perciba su conexión con la causa iniciada o que se prevea incoar (marg. 70). Por ello, no puede decirse que se excluya el procedimiento del pre-trial discovery del ámbito de aplicación del citado Reglamento en todo caso. 